\title{
GENERALIZED FINITE DIFFERENCE METHOD FOR ANOMALOUS DIFFUSION ON SURFACES
}

\author{
ZHUOCHAO TANG ${ }^{1,2}$ \& ZHUOJIA FU ${ }^{1,2,3}$ \\ ${ }^{1}$ Key Laboratory of Coastal Disaster and Defence of Ministry of Education, Hohai University, China. \\ ${ }^{2}$ Center for Numerical Simulation Software in Engineering and Sciences, College of Mechanics and Materials, \\ Hohai University, China. \\ ${ }^{3}$ Institute of Continuum Mechanics, Leibniz University Hannover, Germany.
}

\begin{abstract}
In this study, a localized collocation method called generalized finite difference method (GFDM) is developed to solve the anomalous diffusion problems on surfaces. The expressions of the surface Laplace operator, surface gradient operator and surface divergence operator in tangent space are given explicitly, which is different from the definition of differential operators in the Euclidean space. Based on the moving least square theorem and Taylor series, GFDM shares similar properties with standard FDM and avoids mesh dependence, enabling numerical approximations of the surface operators on complex 3D surfaces. Simultaneously, a standard finite difference scheme is adopted to discretize the time fractional derivatives. By using GFDM, we succeed in solving both constant- and variableorder time fractional diffusion models on surfaces. Numerical examples show that the present meshless scheme has good accuracy and efficiency for various fractional diffusion models.

Keywords: anomalous diffusion, constant- and variable-order time fractional diffusion models, generalized finite difference method, surface PDEs.
\end{abstract}

\section{INTRODUCTION}

Anomalous diffusion phenomenon [1] is widespread in porous media mechanics [2], non-Newtonian fluid mechanics [3], viscoelastic mechanics [4], soft matter mechanics [5] and so on $[6,7]$. Compared with non-linear diffusion models, fractional order diffusion models show better characteristics in describing the anomalous diffusion behaviour, such as less parameters and easier numerical computation. And due to fact of non-local property [8], fractional derivatives can also be used to describe the complex non-Markov systems [9]. Fractional diffusion models, which describe anomalous diffusion behaviour very well, have been studied for many years. Usually, in most cases, it is extremely difficult and even impossible to find analytical solutions to fractional differential equations; so, at present, much work about fractional diffusion is focused on numerical solution in the whole computational space.

However, in recent years, with increasing attractiveness of partial differential equations (PDEs) defined on surfaces or manifolds [10-12], there are only a few reports of fractional order equations defined on surfaces. Surface PDEs or surface operators are a type of PDEs or operators defined in tangent space, which are different in Euclidean space. Also, most existing surface PDE solvers can be classified into two types: the so-called intrinsic methods and embedding methods. The first one attempts to solve PDEs using surface-based meshes and coordinates, while the second one attempts to extend the PDE to the embedding space. Constructing a good meshing of a manifold or surface could be a very difficult process. In this study, we employ a standard finite difference method for temporal discretization and introduce the meshless generalized finite difference method (GFDM) [13] for spatial discretization to solve constant- and variable-order fractional diffusion equations on surfaces.

The history of meshless methods [14-17] dates back to the last century. In meshless methods, a set of nodes are generated instead of meshes. The GFDM adopted in this study is based 
on Taylor series expansions and weighted least-squares method to approximate the derivatives at each computational node by the linear summation of neighbouring nodal values. And then, a sparse linear system is constructed and solved by the standard sparse matrix solver. This method was first proposed by Liszka and Orkisz [18] in 1980s and was gradually improved and perfected until Benito et al. put forward the most advanced version in 2001 [19], including mesh generation, local approximation and automatic selection of nodes. In addition, GFDM, as a localized collocation method, is very suitable for large-scale problems and till now, GFDM has been successfully applied in many areas [20-22].

This paper first applies the GFDM to 3D constant- and variable-order fractional diffusion problems. This paper is organized as follows: methodology, numerical examples and discussions, and conclusions. In Section 2, the surface differential operators and methods referred above are described specifically. In Section 3, several examples and discussion are presented. And lastly, in Section 4, the conclusions are given.

\section{METHODOLOGY}

In this section, we focus our attention on the theory of fractional diffusion model, meshless GFDM and surface differential operators.

\subsection{Time fractional anomalous diffusion model}

Without loss of generality, the constant- and variable-order fractional diffusion equation defined on surfaces can be followed by the equation

$$
\frac{\partial^{a(t)} u(\mathbf{x}, t)}{\partial t^{a(t)}}=\left(D \Delta_{s}+\vec{v} \cdot \nabla_{s}-\lambda\right) u(\mathbf{x}, t)+Q(\mathbf{x}, t), 0<\alpha(t)<1, \mathbf{x} \in \Gamma_{s}, t \in(0, T),
$$

with the initial condition

$$
u(\mathbf{x}, 0)=u_{0}(\mathbf{x}), \mathbf{x} \in \Gamma_{s}
$$

where $\Delta_{s}$ and $\nabla_{s}$ are the Laplace-Beltrami operator and the surface gradient operator, respectively; $D, \lambda \vec{v}$ are the diffusion coefficient, reaction coefficient and velocity vector, respectively; $Q(\mathbf{x}, t)$.and $u_{0}(\mathbf{x})$ are the known functions; $T$ is the total time to be considered and $\left(\partial^{\alpha(t)} / \partial t^{\alpha(t)}\right)$ is the variable-order time fractional derivative of the order $\alpha(t)$ with respect

The variable-order time fractional derivative mentioned above can be defined by the equation

$$
\frac{\partial^{a(t)} u(\mathbf{x}, t)}{\partial t^{a(t)}}=\int_{0}^{t} \frac{1}{\Gamma(1-a(\eta))} \frac{\partial u(\mathbf{x}, \eta)}{\partial \eta} \frac{d \eta}{(t-\eta)^{a(t)}}, 0<a(t)<1
$$

When $a(t)$ is a constant or independent of $t$, eqn (3) can be simplified to the following famous Caputo constant-order time fractional derivative:

$$
\frac{\partial^{a(t)} u(\mathbf{x}, t)}{\partial t^{a(t)}}=\frac{1}{\Gamma(1-a)} \int_{0}^{t} \frac{\partial u(\mathbf{x}, \eta)}{\partial \eta} \frac{d \eta}{(t-\eta)^{a}}, 0<a<1,
$$

in which $\Gamma$ is the gamma function. In a way, eqn (4) is a special case of eqn (3). 
By using standard FDM, the temporal fractional derivative can be discretized as

$$
\begin{aligned}
& D \theta \Delta_{s} u^{k+1}+\theta \vec{v} \cdot \nabla_{s} u^{k+1}-\left(\lambda \theta+a_{0}\right) u^{k+1} \\
& =\left\{\begin{array}{lc}
-\theta Q^{k+1}-(1-\theta)\left(D \Delta_{s} u^{k}+\vec{v} \cdot \nabla_{s} u^{k}+Q^{k}-\lambda u^{k}\right)-a_{0} u^{k}+\sum_{j=1}^{k} a_{j} b_{j}\left(u^{k-j+1}-u^{k-j}\right), & k \geq 1 \\
-\theta Q^{1}-(1-\theta)\left(D \Delta_{s} u^{0}+\vec{v} \cdot \nabla_{s} u^{0}+Q^{0}-\lambda u^{0}\right)-a_{0} u^{0}, & k=0
\end{array},\right.
\end{aligned}
$$

where $a_{j}, b_{j}$ are known coefficients. More details on temporal discretization could be found in [23].

\subsection{Time fractional anomalous diffusion model}

In general, GFDM is an extension of standard FDM; so, the resulting expression is similar, which is as follows:

$$
\begin{gathered}
\left.\frac{\partial u}{\partial x_{k}}\right|_{i}=w_{i}^{x_{k}, i} u_{i}+\sum_{j=1, j \neq i}^{n s} w_{j}^{x_{k}, i} u_{j}, \quad \mathbf{x}_{i}=\xi \in \Xi, k=1,2, \cdots, d, \\
\left.\frac{\partial^{2} u}{\partial x_{k} \partial x_{k^{\prime \prime}}}\right|_{i}=w_{i}^{x_{k} x_{k^{\prime \prime}}, i} u_{i}+\sum_{j=1, j \neq i}^{n s} w_{j}^{x_{k} x_{k^{\prime \prime}}, i} u_{j}, \mathbf{x}_{i}=\xi \in \Xi, k=1,2, \cdots, d, k^{\prime \prime}=k, k+1, \cdots, d,
\end{gathered}
$$

For better understanding, two sketches including 2D and 3D are shown in Fig.1.

For a given central node (both including interior and boundary nodes) in the subdomain, Taylor series is used to construct the numerical differential scheme:

$$
u\left(\mathbf{x}_{j}\right)=u(\xi)+\sum_{k=1}^{d} h_{j k} \frac{\partial u(\xi)}{\partial x_{k}}+\frac{1}{2}\left(\sum_{k=1}^{d} h_{j k} \frac{\partial}{\partial x_{k}}\right)^{2} u(\xi)+o\left(\rho^{3}\right),
$$

where $h_{j k}=x_{j k}-\xi_{k}, \rho_{j}=\sqrt{\sum_{k=1}^{d} h_{j k}^{2}}$. Considering the remainder term of eqn (8), the residual function $B(u)$ is defined as

$$
B(u)=\sum_{j=1}^{n s}\left[\left(u(\xi)-u\left(\mathbf{x}_{j}\right)+\sum_{k=1}^{d} h_{j k} \frac{\partial u(\xi)}{\partial x_{k}}+\frac{1}{2}\left(\sum_{k=1}^{d} h_{j k} \frac{\partial}{\partial x_{k}}\right)^{2} u(\xi)\right) \beta\left(\rho_{j}\right)\right]^{2} .
$$

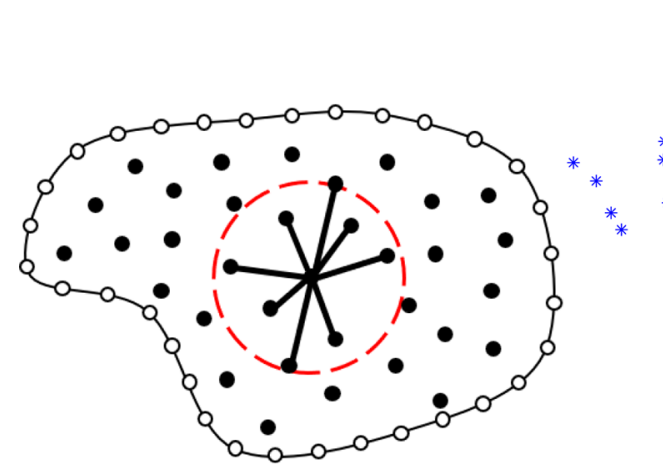

(a)

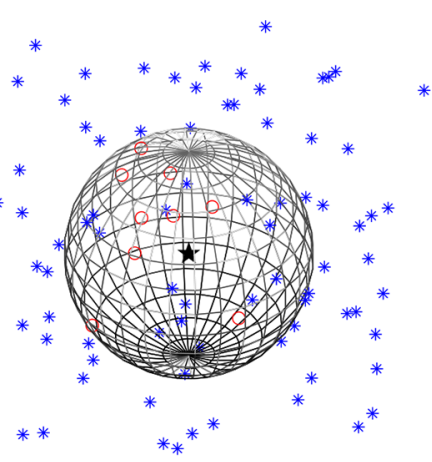

(b)

Figure 1: Sketch of the nodes distribution and subdomain: (a) 2D and (b) 3D. 
The weight function $\beta\left(\rho_{j}\right)$ used in this study is

$$
\beta\left(\rho_{j}\right)=\left\{\begin{array}{c}
\left(\rho_{j}\right)^{-3}, \rho_{j} \leq d_{j} \\
0, \rho_{j}>d_{j}
\end{array}\right.
$$

It is noted that this method requires minimizing eqn (9), after which we could obtain a linear system

$$
\mathbf{A D}_{u}=\mathbf{b}
$$

where

$$
\begin{aligned}
& \mathbf{A}=\left[\begin{array}{cc}
{\left[\sum_{j=1}^{n s} h_{j k} h_{j \bar{k}} \beta^{2}\right]_{d \times d}} & {\left[\sum_{j=1}^{n s} a\left(\bar{k}, k^{\prime}\right) h_{j k} h_{j \bar{k}} h_{j k^{\prime}} \beta^{2}\right]_{d \times\left(n_{v}-d\right)}} \\
S Y M & {\left[\sum_{j=1}^{n s}\left(a\left(k, k^{\prime \prime}\right) h_{j k} h_{j k^{\prime \prime}}\right)\left(a\left(\bar{k}, k^{\prime}\right) h_{j \bar{k}} h_{j k^{\prime}}\right) \beta^{2}\right]_{\left(n_{v}-d\right) \times\left(n_{v}-d\right)}}
\end{array}\right], \\
& \mathbf{b}=\left[\begin{array}{c}
\sum_{j=1}^{n s}\left(-u(\xi)+u\left(\mathbf{x}_{j}\right)\right) h_{j k} \beta^{2} \\
\vdots \\
\sum_{j=1}^{n s}\left(-u(\xi)+u\left(\mathbf{x}_{j}\right)\right) a\left(k, k^{\prime \prime}\right) h_{j k} h_{j k} \beta^{2}
\end{array}\right]_{n_{v} \times 1}=\mathbf{B}_{n_{v} \times N} \mathbf{U}=\mathbf{B}\left[\begin{array}{c}
u\left(\mathbf{x}_{1}\right) \\
u\left(\mathbf{x}_{2}\right) \\
\vdots \\
u(\xi) \\
\vdots \\
u\left(\mathbf{x}_{N}\right)
\end{array}\right],
\end{aligned}
$$

in which $k=1,2, \cdots, d ; \bar{k}=1,2, \cdots, d ; k^{\prime}=\bar{k}, \bar{k}+1, \cdots, d ; k^{\prime \prime}=k, k+1, \cdots, d, n s$ is the number of the neighbouring nodes and

$$
a\left(k, k^{\prime \prime}\right)= \begin{cases}1 / 2, & k=k^{\prime \prime} \\ 1, & k \neq k^{\prime \prime}\end{cases}
$$

\subsection{Surface differential operators}

The surface gradient operator is an extension of standard gradient operator from the Euclidean space, which could be given as

$$
\nabla_{s}:=\mathbf{p} \nabla
$$

in which $\mathbf{p}(\mathbf{x})=\left[\vec{p}_{1}, \vec{p}_{1}, \ldots \vec{p}_{d}\right](\mathbf{x}):=\mathbf{I}_{d}-\mathbf{n n}^{T}$ and $\mathbf{I}_{d}$ is the $d \times d$ identity matrix.

Similarly, the Laplace-Beltrami operator is given by

$$
\Delta_{s}:=\nabla_{s} \cdot \nabla_{s}
$$

A significant relation between surface operators and Euclidean operators for any sufficiently smooth function could be found in [24]:

$$
\nabla_{s} u:=\nabla u-\mathbf{n} \partial_{\mathbf{n}} u \quad, \quad \Delta_{s} u:=\Delta u-H_{s} \partial_{\mathbf{n}} u-\partial_{\mathbf{n}}{ }^{(2)} u .
$$


where $\nabla_{s} u:=\nabla u-\mathbf{n} \partial_{\mathbf{n}} u, \quad \Delta_{s} u:=\Delta u-H_{s} \partial_{\mathbf{n}} u-\partial_{\mathbf{n}}{ }^{(2)} u$, and $J$ is the Jacobian operator in Euclidean space.

Once the relationship between surface operators and Euclidean operators is given explicitly, the numerical expressions could be found by using eqns (6) and (7).

\section{NUMERICAL EXAMPLES AND DISCUSSION}

In this section, three examples are provided including the single and coupled constant-order and the coupled variable-order fractional diffusion models. To quantify the accuracy and effectiveness of our numerical solutions, we introduce the root mean square error (RMSE) measures as follows:

$$
\operatorname{RSME}\left(u_{i}(\mathbf{x}, t)\right)=\sqrt{\frac{1}{N} \sum_{j=1}^{N}\left[u\left(\mathbf{x}_{j}, t\right)-\tilde{u}\left(\mathbf{x}_{j}, t\right)\right]^{2}}
$$

wherein $N$ is the total number of discretization nodes and $\tilde{u}\left(\mathbf{x}_{j}, t\right), u\left(\mathbf{x}_{j}, t\right)$ stand for the numerical solution and analytical solution, respectively.

\subsection{Example 1: single and coupled constant-order fractional diffusion models}

3.1.1 Single diffusion equation on the sphere

In this example, the governing equation is given by

$$
\frac{\partial^{a} u(\mathbf{x}, t)}{\partial t^{a}}=\delta_{u} \Delta_{S} u(\mathbf{x}, t)+Q(\mathbf{x}, t), 0<\alpha<1, \mathbf{x} \in \Gamma, t \in(0, T),
$$

wherein $a=\frac{1}{2}$ and $\delta_{u}=1$. The exact solution is expressed as

$$
u(t, \mathbf{x})=x^{3} \cdot t+y^{3}+2 z^{3}
$$

In this test example, three kinds of time integration methods including explicit time integral method $(\theta=0)$, implicit time integral method $(\theta=1)$ and Crank-Nicholson method $(\theta=0.5)$ are considered. But it has been proved that explicit time integral method is usually limited in the criterion between time step and space step, which leads to poor accuracy and efficiency. Therefore, the (1) implicit time integral method and the (2) Crank-Nicholson method are adopted in this example.

It is easy to find from Fig. 2 that the GFDM combined with implicit time integral method $(\theta=1)$ and with Crank-Nicholson method $(\theta=0.5)$ both show better stability and accuracy. However, from eqn (5), we could find if $\theta=0.5$, the computation is more than the case when $\theta=1$. Therefore, implicit time integral method is chosen in subsequent examples. Also, in this test, the effect of the number of neighbouring nodes is taken into consideration. Usually, the number of adjacent nodes should be more than twice the number of terms in the Taylor expansion. To deal with more complex surfaces, we will take 50 neighbouring points by default to ensure its accuracy and stability.

\subsubsection{Coupled diffusion equations on the complex surfaces}

In this test, we expand single diffusion equations to coupled diffusion equations on the complex surfaces. The governing equation is given as 


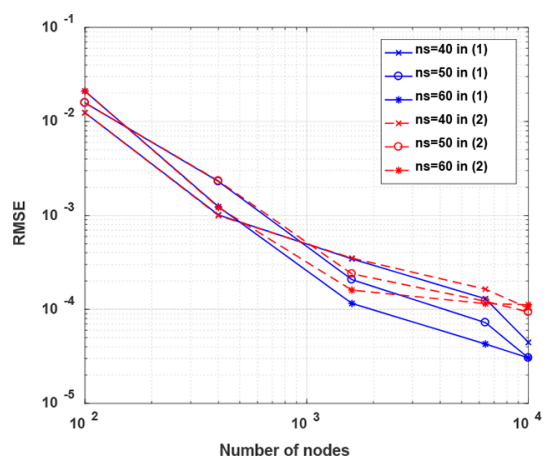

(a)

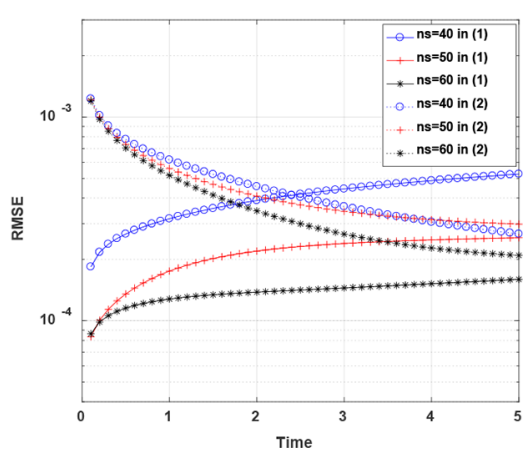

(b)

Figure 2: Convergence results against the number of nodes (a) and time (b) on sphere.

$$
\left\{\begin{array}{l}
\frac{\partial^{a} u(\mathbf{x}, t)}{\partial t^{a}}=\delta_{u} \Delta_{S} u(\mathbf{x}, t)+v+Q_{1}(\mathbf{x}, t) \\
\frac{\partial^{a} v(\mathbf{x}, t)}{\partial t^{a}}=\delta_{v} \Delta_{S} v(\mathbf{x}, t)+u+Q_{2}(\mathbf{x}, t)
\end{array}, 0<a<1, \mathbf{x} \in \Gamma, t \in(0, T)\right.
$$

wherein $\alpha=0.5$ and $\delta_{u}=\delta_{v}=1$, and the exact solution is given as

$$
\left\{\begin{array}{l}
u(t, \mathbf{x})=x^{3} \cdot t+y^{3}+2 z^{3} \\
u(t, \mathbf{x})=x^{3} \cdot t+2 y^{3}+z^{3}
\end{array}\right.
$$

Figure 3 shows the distribution of absolute error under red blood cell shape, torus shape and tumour shape. Table 2 gives more results on complex surfaces such as Bean, Bretzel, Cross and DC surfaces. Although the accuracy of the GFDM in solving fractional PDEs with complex solutions is less, we could still conclude that GFDM combined with implicit time integration method possesses a good accuracy and stability in solving single constant-order fractional PDE.

\subsection{Example 2: coupled variable-order fractional diffusion models on surfaces}

Similarly, we changed constant-order fractional PDEs into variable-order fractional PDEs and the governing equation is given as

$$
\left\{\begin{array}{l}
\frac{\partial^{a(t)} u(\mathbf{x}, t)}{\partial t^{a(t)}}=\delta_{u} \Delta_{S} u(\mathbf{x}, t)+v+Q_{1}(\mathbf{x}, t) \\
\frac{\partial^{a(t)} v(\mathbf{x}, t)}{\partial t^{a(t)}}=\delta_{v} \Delta_{S} v(\mathbf{x}, t)+u+Q_{2}(\mathbf{x}, t)
\end{array}, 0<a<1, \mathbf{x} \in \Gamma, t \in(0, T),\right.
$$

wherein $a=0.8+0.2 t / T, T=0.2, \delta_{u}=\delta_{v}=1$, and the exact solution is given as

$$
\left\{\begin{array}{l}
u(t, \mathbf{x})=x^{3} \cdot t+y^{3}+2 z^{3} \\
u(t, \mathbf{x})=x^{3} \cdot t+2 y^{3}+z^{3}
\end{array} .\right.
$$




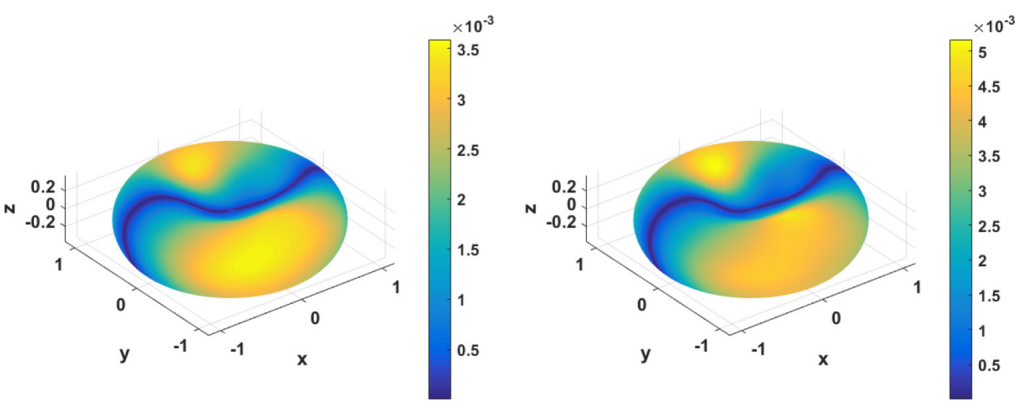

(a)
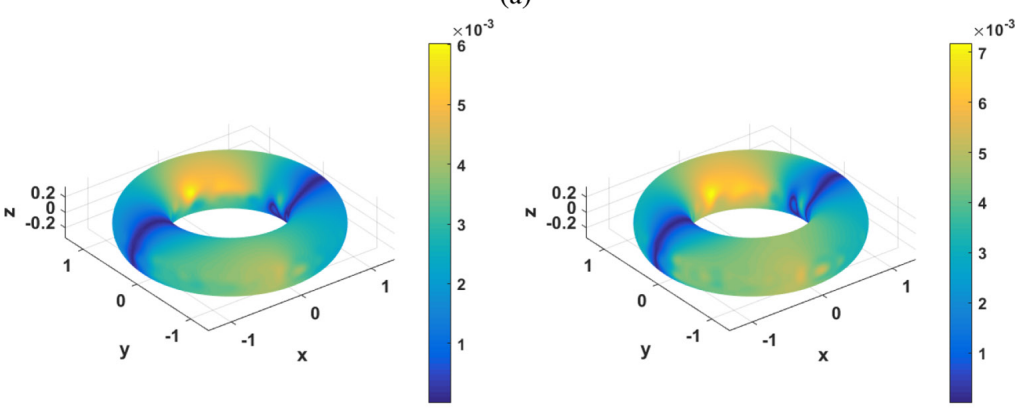

(b)
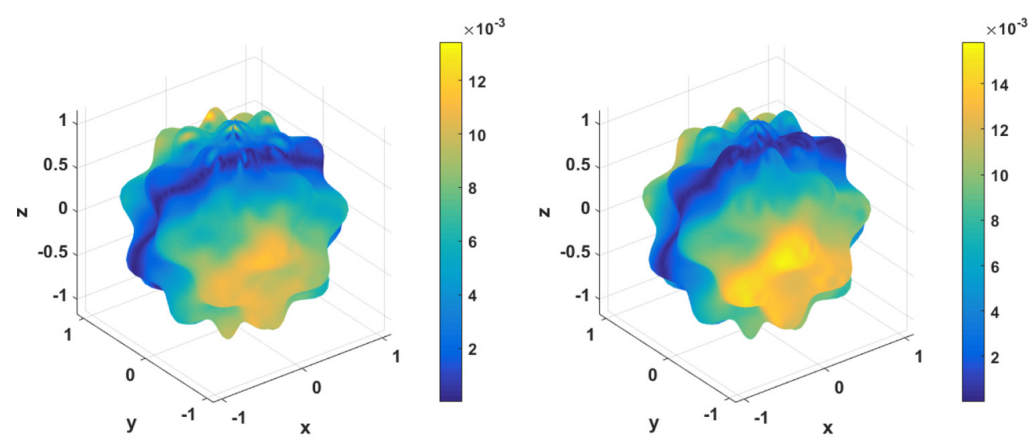

(c)

Figure 3: Distribution of absolute error $u$ (left) and $v$ (right) at $t=0.5$ on (a) RBC, (b) torus and (c) tumour.

Table 1: RMSE on different surfaces at $\mathrm{t}=0.5$.

\begin{tabular}{lllllllll}
\hline Shape & Sphere & Bean & Bretzel & Cross & DC & RBC & Torus & Tumour \\
\hline N & 2500 & 3600 & 3974 & 2010 & 5024 & 4096 & 5256 & 3600 \\
RMSE(u) & $1.43 \mathrm{E}-$ & $9.74 \mathrm{E}-$ & $8.87 \mathrm{E}-$ & $3.90 \mathrm{E}-$ & $2.75 \mathrm{E}-$ & $3.46 \mathrm{E}-$ & $3.36 \mathrm{E}-$ & $4.84 \mathrm{E}-$ \\
& 04 & 04 & 03 & 03 & 03 & 03 & 03 & 03 \\
$\mathrm{RMSE}(\mathrm{v})$ & $1.21 \mathrm{E}-$ & $2.10 \mathrm{E}-$ & $6.70 \mathrm{E}-$ & $2.86 \mathrm{E}-$ & $1.78 \mathrm{E}-$ & $2.62 \mathrm{E}-$ & $2.37 \mathrm{E}-$ & $8.29 \mathrm{E}-$ \\
& 04 & 03 & 03 & 03 & 03 & 03 & 03 & 03 \\
\hline
\end{tabular}




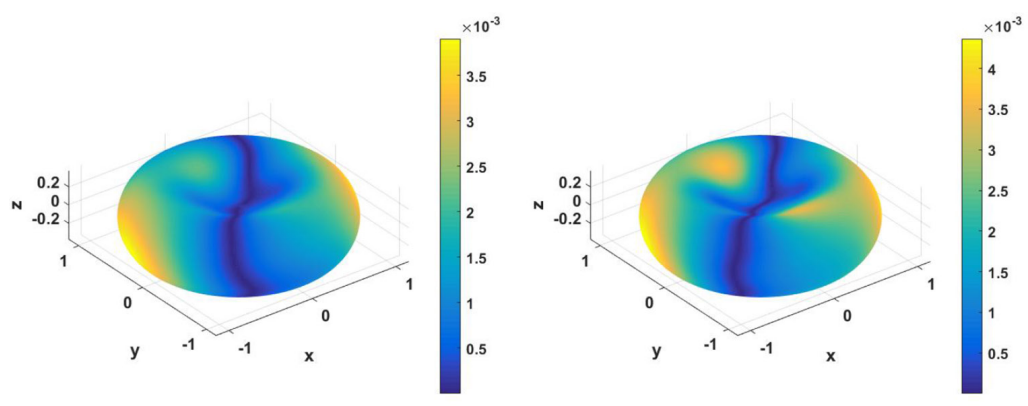

(a)
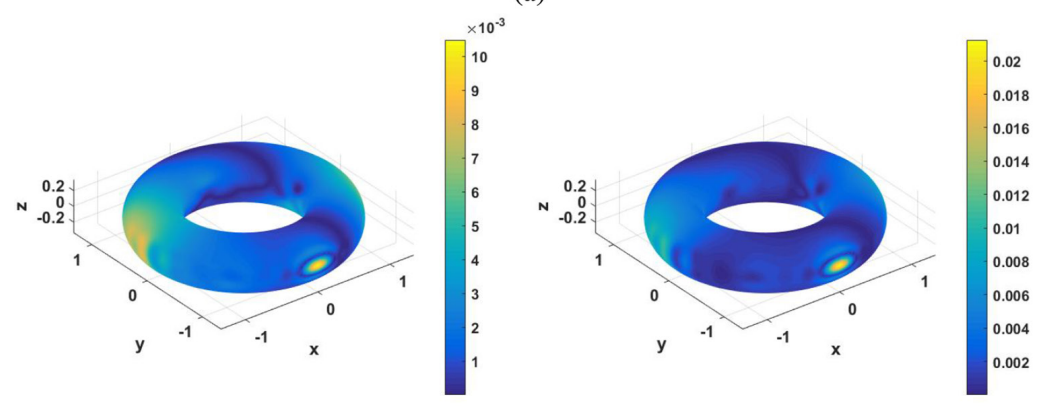

(b)
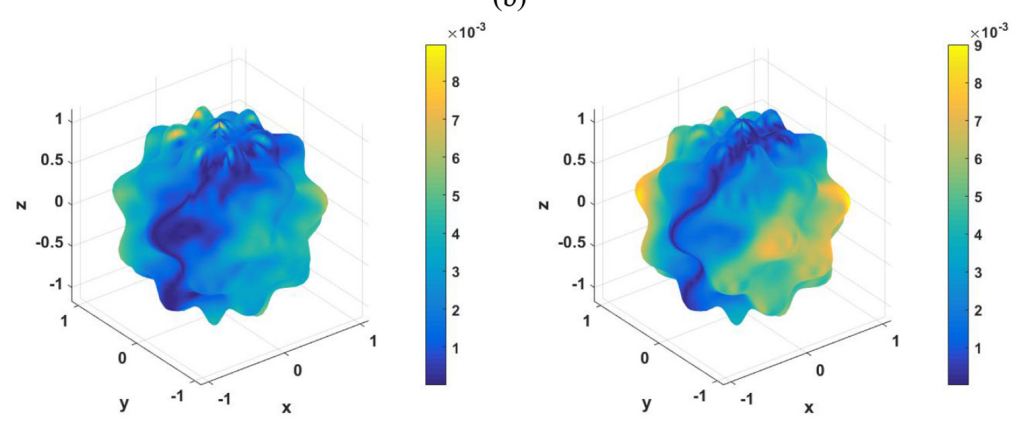

(c)

Figure 4: Distribution of absolute error $u$ (left) and $v$ (right) at $\mathrm{t}=0.2$ on (a) RBC, (b) torus and (c) tumour.

Figure 4 shows the distribution of absolute error under red blood cell shape, torus shape and tumour shape. Table 2 gives the RMSE under different complex surfaces. Figure 4 and Table 2 show that the accuracy of GFDM in solving coupled variable-order fractional diffusion equations on surfaces is good. Compared with constant-order fractional PDEs on surfaces, variable-order fractional PDEs exhibit stronger non-linearity, which shows that the promoted method has a good robustness.

\section{CONCLUSION}

In this study, the GFDM was developed to solve constant- and variable-order fractional diffusion equations on 3D complex surfaces. Due to the explicit relationship between surface 
Table 2: RMSE on different surfaces at $\mathrm{t}=0.2$.

\begin{tabular}{lllllllll}
\hline Shape & Sphere & Bean & Bretzel & Cross & DC & RBC & Torus & Tumour \\
\hline N & 4096 & 3600 & 8906 & 2010 & 5024 & 4096 & 5256 & 3600 \\
RMSE(u) & $1.00 \mathrm{E}-$ & $1.00 \mathrm{E}-$ & $1.00 \mathrm{E}-$ & $3.10 \mathrm{E}-$ & $4.20 \mathrm{E}-$ & $2.70 \mathrm{E}-$ & $3.00 \mathrm{E}-$ & $2.00 \mathrm{E}-$ \\
& 03 & 03 & 03 & 03 & 03 & 03 & 03 & 03 \\
& & & & & & \\
RMSE(v) & $1.00 \mathrm{E}-$ & $1.90 \mathrm{E}-$ & $1.90 \mathrm{E}-$ & $1.90 \mathrm{E}-$ & $2.30 \mathrm{E}-$ & $1.70 \mathrm{E}-$ & $1.70 \mathrm{E}-$ & $3.70 \mathrm{E}-$ \\
& 03 & 03 & 03 & 03 & 03 & 03 & 03 & 03 \\
\hline
\end{tabular}

operators and Euclidean operators, the surface operators can be approximated directly by GFDM. This method is different from other embedding methods in that it only requires 'scattered' nodes on the surface and the corresponding normal vectors. Three examples including single and coupled constant- and variable-order fractional diffusion equations on 3D complex surfaces were provided to verify the accuracy, effectiveness and robustness of the promoted method.

\section{ACKNOWLEDGEMENTS}

The work described in this paper was supported by the National Science Fund of China (Grant No. 11772119), the Fundamental Research Funds for the Central Universities (Grant No. B200202124), the Foundation for Open Project of State Key Laboratory of Mechanics and Control of Mechanical Structures (Nanjing University of Aeronautics And Astronautics) (Grant No. MCMS-E-0519G01), Alexander von Humboldt Research Fellowship (ID: 1195938) and the Six Talent Peaks Project in Jiangsu Province of China (Grant No. 2019-KTHY-009).

\section{APPENDIX}

The 3D surfaces used above are described as follows:

Sphere: $S=x^{2}+y^{2}+z^{2}-1$

RBC: $S=\left\{\begin{array}{l}x=1.15 \cos (\lambda) \cos (\theta) \\ y=1.15 \sin (\lambda) \cos (\theta) \\ z=0.5 \sin (\lambda)\left(0.24+2.3 \cos (\theta)^{2}-1.3 \cos (\theta)^{4}\right)\end{array},-\pi \leq \lambda \leq \pi,-\frac{\pi}{2} \leq \theta \leq \frac{\pi}{2}\right.$

Tours: $S=\left(1-\sqrt{x^{2}+y^{2}}\right)^{2}+z^{2}-\frac{1}{9}$

Bean: $S=\frac{(x+0.6 \cos (\pi z / 2))^{2}}{(0.64(1-0.4 \cos (\pi z / 2)))}+\frac{y^{2}}{(0.64(1-0.1 \cos (\pi z / 2)))}+z^{2}-4$

Bretzel: $S=\left(x^{2}\left(1-x^{2}\right)-y^{2}\right)^{2}+\frac{1}{2} z^{2}-\frac{1}{40}$

Cross: $S=\sqrt{(x-1)^{2}+y^{2}+z^{2}} \sqrt{(x+1)^{2}+y^{2}+z^{2}} \sqrt{x^{2}+(y-1)^{2}+z^{2}} \sqrt{x^{2}+(y+1)^{2}+z^{2}}-1.1$

DC: $S=\left(x^{2}+y^{2}+z^{2}+2.61\right)^{2}-4(2 x+0.6245)^{2}-14.44 y^{2}$ 
Tumour: $S=\left\{\begin{aligned} x & =\left(1+\frac{1}{6} \sin (6 \lambda) \sin (7 \theta)\right) \cos (\lambda) \cos (\theta) \\ y & =\left(1+\frac{1}{6} \sin (6 \lambda) \sin (7 \theta)\right) \sin (\lambda) \cos (\theta),-\pi \leq \lambda \leq \pi,-\frac{\pi}{2} \leq \theta \leq \frac{\pi}{2} \\ z & =\left(1+\frac{1}{6} \sin (6 \lambda) \sin (7 \theta)\right) \sin (\theta)\end{aligned}\right.$

\section{REFERENCES}

[1] Sun, H.G., Chen, W. \& Chen, Y., Variable-order fractional differential operators in anomalous diffusion modeling. Physica A Statistical Mechanics \& Its Applications, 388, pp. 4586-4592, 2009. https://doi.org/10.1016/j.physa.2009.07.024

[2] Koch, D.L. \& Brady, J.F., Anomalous diffusion in heterogeneous porous media. Physics of Fluids, 31(5), pp. 965-973, 1988. https://doi.org/10.1063/1.866716

[3] Ezzat, M.A., Thermoelectric MHD non-Newtonian fluid with fractional derivative heat transfer. Physica B Condensed Matter, 405(19), pp. 4188-4194, 2010. https://doi.org/ 10.1016/j.physb.2010.07.009

[4] Welch, S.W.J., Rorrer, R.A.L. \& Duren, R.G., Application of time-based fractional calculus methods to viscoelastic creep and stress relaxation of materials. Mechanics of Time-Dependent Materials, 3(3), pp. 279-303, 1999. https://doi.org/10.1023/a: 1009834317545

[5] Chen, W., Mesoscopic fractional quantum in soft matter. arXiv preprint quant-ph/ $0505050,2005$.

[6] Sun, H., Chen, W., Li, C. \& Chen, Y., Fractional differential models for anomalous diffusion. Physica A: Statistical Mechanics and its Applications, 389(14), pp. 2719-2724, 2010. https://doi.org/10.1016/j.physa.2010.02.030

[7] Chen, W., Sun, H., Zhang, X. \& Korošak, D., Anomalous diffusion modeling by fractal and fractional derivatives. Computers \& Mathematics with Applications, 59(5), pp. 1754-1758, 2010. https://doi.org/10.1016/j.camwa.2009.08.020

[8] Atangana, A. \& Baleanu, D., New fractional derivatives with nonlocal and non-singular kernel: theory and application to heat transfer model. arXiv preprint arXiv:1602.03408, 2016.

[9] Malaiya, Y.K. \& Su, S.Y., Analysis of an important class of non-Markov systems. IEEE Transactions on Reliability, 31(1), pp. 64-68, 1982. https://doi.org/10.1109/tr.1982. 5221231

[10] Fuselier, E.J. \& Wright, G.B., A high-order kernel method for diffusion and reactiondiffusion equations on surfaces. Journal of Scientific Computing, 56(3), pp. 535-565, 2013. https://doi.org/10.1007/s10915-013-9688-x

[11] Petras, A., Ling, L., Piret, C. \& Ruuth, S.J., A least-squares implicit RBF-FD closest point method and applications to PDEs on moving surfaces. Journal of Computational Physics, 381, pp. 146-161, 2019. https://doi.org/10.1016/j.jcp.2018.12.031

[12] Petras, A., Ling, L. \& Ruuth, S.J., An RBF-FD closest point method for solving PDEs on surfaces. Journal of Computational Physics, 370, pp. 43-57, 20118. https://doi.org/ 10.1016/j.jcp.2018.05.022

[13] Fu, Z.J., Tang, Z.C., Zhao, H.T., Li, P.W. \& Rabczuk, T., Numerical solutions of the coupled unsteady nonlinear convection-diffusion equations based on generalized finite difference method. The European Physical Journal Plus, 134(6), 272, 2019. https://doi. org/10.1140/epjp/i2019-12786-7 
[14] Fu, Z.J., Chen, W. \& Gu, Y., Burton-Miller-type singular boundary method for acoustic radiation and scattering. Journal of Sound and Vibration, 333(16), pp. 3776-3793, 2014. https://doi.org/10.1016/j.jsv.2014.04.025

[15] Fu, Z.J., Chen, W., Lin, J. \& Cheng, A.H.D., Singular boundary method for various exterior wave applications. International Journal of Computational Methods, 12(2), 1550011, 2015. https://doi.org/10.1142/s0219876215500115

[16] Gu, Y., Chen, W. \& Zhang, J., Investigation on near-boundary solutions by singular boundary method. Engineering Analysis with Boundary Elements, 36(8), pp. 1173-1182, 2012. https://doi.org/10.1016/j.enganabound.2012.01.006

[17] Tang, Z.C., Fu, Z.J., Zheng, D.J. \& Huang, J.D., Singular boundary method to simulate scattering of SH wave by the canyon topography. Adv Appl Math Mech, 10(4), pp. 912-924, 2018. https://doi.org/10.4208/aamm.oa-2017-0301

[18] Liszka, T. \& Orkisz, J., Finite difference method at arbitrary irregular meshes in nonlinear problems of applied mechanics. In Structural mechanics in reactor technology, 1977.

[19] Benito, J.J., Urena, F. \& Gavete, L., Solving parabolic and hyperbolic equations by the generalized finite difference method. Journal of Computational and Applied Mathematics, 209(2), pp. 208-233, 2007. https://doi.org/10.1016/j.cam.2006.10.090

[20] Fan, C.M., Chu, C.N., Šarler, B. \& Li, T.H., Numerical solutions of waves-current interactions by generalized finite difference method. Engineering Analysis with Boundary Elements, 100, pp. 150-163, 2019. https://doi.org/10.1016/j.enganabound.2018.01.010

[21] Fu, Z.J., Xie, Z.Y., Ji, S.Y., Tsai, C.C. \& Li, A.L., Meshless generalized finite difference method for water wave interactions with multiple-bottom-seated-cylinder-array structures. Ocean Engineering, 195, 106736, 2020.

[22] Qu, W., Gu, Y., Zhang, Y., Fan, C.M. \& Zhang, C., A combined scheme of generalized finite difference method and Krylov deferred correction technique for highly accurate solution of transient heat conduction problems. International Journal for Numerical Methods in Engineering, 117(1), pp. 63-83, 2019. https://doi.org/10.1002/nme.5948

[23] Fu, Z.J., Chen, W. \& Ling, L., Method of approximate particular solutions for constantand variable-order fractional diffusion models. Engineering Analysis with Boundary Elements, 57, pp. 37-46, 2015. https://doi.org/10.1016/j.enganabound.2014.09.003

[24] Cheung, K.C. \& Ling, L., A kernel-based embedding method and convergence analysis for surfaces pdes. Siam Journal on Scientific Computing, 40(1), pp. A266-A287, 2018. https://doi.org/10.1137/16m1080410 